\title{
Cervical Spondylotic Myelopathy with Ossification of Posterior Longitudinal Ligament: Which Is the Most Suitable Surgical Procedure? A Technical Note
}

\author{
Edvin Zekaj ${ }^{1)}$, Christian Saleh ${ }^{2)}$, Andrea Franzini' ${ }^{3)}$, Andrea Ciuffi ${ }^{3)}$ and Domenico Servello ${ }^{1)}$ \\ 1) Department of Neurosurgery, IRCCS Istituto Ortopedico Galeazzi, Milan, Italy \\ 2) Department of Neurophysiology and Neurology, University Hospital Basel, Basel, Switzerland \\ 3) Department of Neurosurgery, Università degli studi di Milano, Milan, Italy
}

\begin{abstract}
:
Introduction: Anterior cervical discectomy and fusion (ACDF) and anterior cervical corpectomy and fusion (ACCF) are widely performed to resolve anterior cervical spine compression. The main purpose of the different surgical techniques is to obtain an adequate decompression of the spinal cord and nerve roots, preserving spinal stability like in oblique corpectomy or leading to a final solid construct to achieve arthrodesis.

Technical Note: We describe a surgical procedure for treating cervical spondylotic myelopathy(CSM) with ossification of the posterior longitudinal ligament (OPLL) at the level of C3-C4 and C4-C5. A double level discectomy C3-C4 and C4-C5 and selective posterior wedge corpectomy of C3, C4, and C5 were performed. Two cages (Zero-P VA) at C3-C4 and C4-C5 were positioned to obtain segmental stability and arthrodesis. An extended anterior cervical canal decompression was obtained and confirmed by postsurgical CT scan. At 15 months, dynamic X-ray showed fusion, and cervical magnetic resonance imaging (MRI) showed evidence of spinal canal decompression.

Conclusions: Anterior cervical discectomy followed by selective wedge corpectomy appears to be a safe and effective technique for anterior spinal cord compression extending above and below the intervertebral disc space.
\end{abstract}

Keywords:

Anterior cervical discectomy, selective wedge corpectomy, anterior spinal cord compression

Spine Surg Relat Res 2021; 5(1): 41-45 dx.doi.org/10.22603/ssrr.2019-0107

\section{Introduction}

Cervical spondylotic myelopathy (CSM) is a fearsome disorder, especially in the elderly ${ }^{1)}$. CSM is a neurological syndrome that results from the acute or chronic compression of the cervical spinal cord due to degenerative processes. Clinical manifestations of CSM range from subacute to acute and chronic fashion. The clinical hallmarks are pyramidal tract and radicular symptoms and signs. Sphincter dysfunction, like micturition urgency or urinary retention, is also frequently present. Posterior tract alterations occur to a lesser degree. If foraminal stenosis is present, patients usually complain of radicular pain, paresthesia, and, in severe cases, paresis with atrophy in the upper limbs ${ }^{2}$. Ossification of the posterior longitudinal ligament (OPLL) of the cervical tract consists of calcification of the posterior longitudinal ligament in his spinal segment, leading to a stenosis of the spinal canal, which may cause clinical symptoms secondary to myelopathy ${ }^{3)}$.

Once CSM or myelopathy is suspected, radiological diagnostic is necessary. Gold standard imaging consists in cervical magnetic resonance imaging (MRI). In selected patients, cervical CT scan is necessary to carefully evaluate osteophyte formations and their cranio-caudal and anteroposterior extensions. OPLL is also evaluated more accurately with CT imaging. Dynamic flexion-extension X-rays may be necessary in patients with suspected instability. Furthermore, surgery is mandatory in patients with CSM secondary to acute compression or in patients with progressions of known CSM. Once surgical treatment is chosen over conservative management, the main challenge is to decide for the most suitable surgical approach. Authors have described several

Corresponding author: Edvin Zekaj, ezekaj@yahoo.com

Received: November 8, 2019, Accepted: April 17, 2020, Advance Publication: June 18, 2020

Copyright (C) 2021 The Japanese Society for Spine Surgery and Related Research 

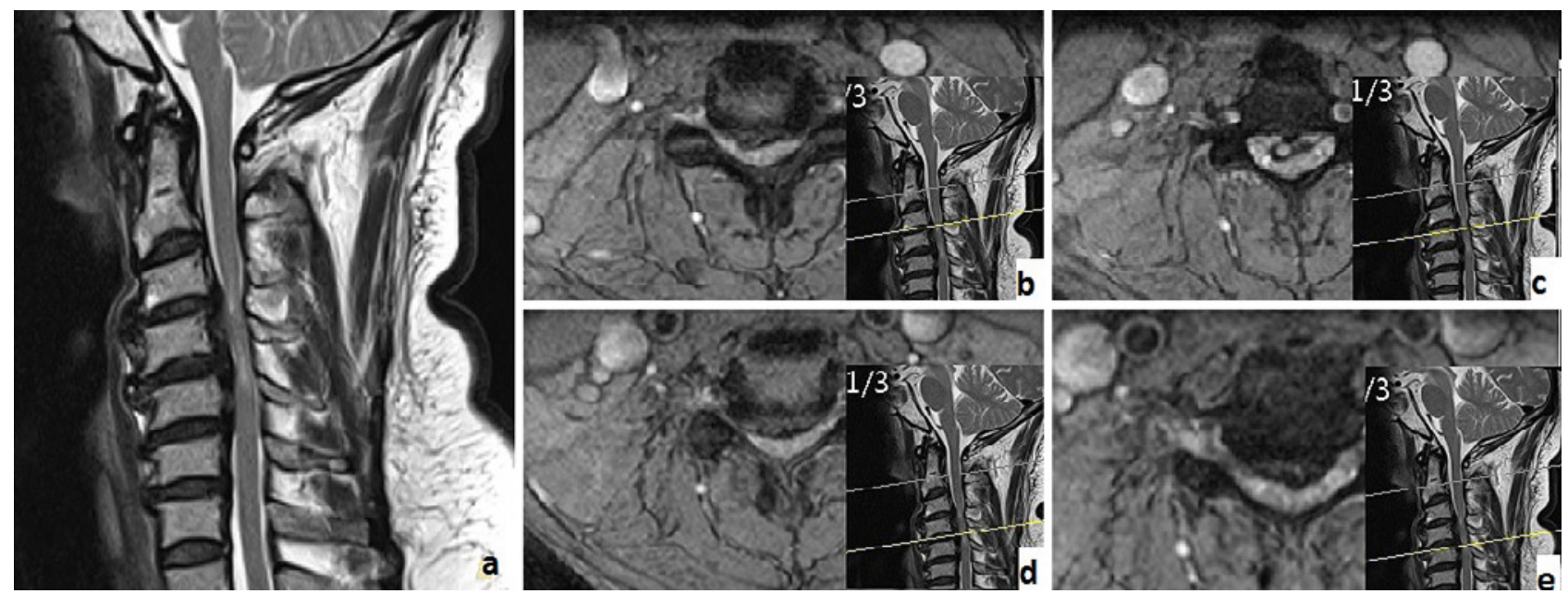

Figure 1. a. Sagittal T2 MRI images showing a severe narrowing of spinal canal at C3-C4 and C4-C5 with spinal cord lesion. b, c, d, e. Axial T2 MRI images showing the severe anterior compression at each level.
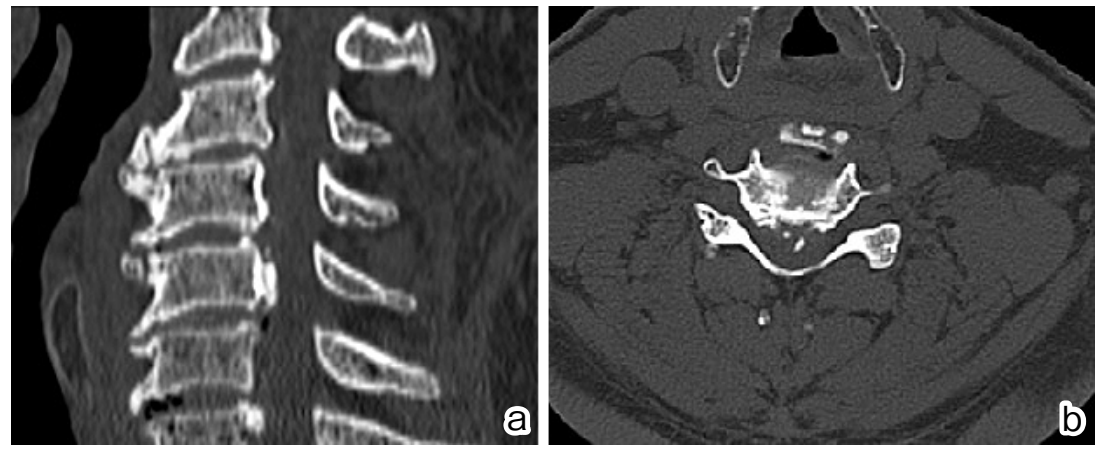

Figure 2. a. Sagittal reconstruction of CT scan showing ossifications of the posterior longitudinal ligament. b. Axial CT scan images showing the extent of spinal canal stenosis.

anterior and posterior procedures in order to relieve spinal cord and radicular compression while ensuring postsurgical spinal stability ${ }^{4}$.

In this paper, we describe a modified corpectomy in a patient with CSM secondary to spondylosis and OPLL extending behind vertebral bodies of $\mathrm{C} 4$ and $\mathrm{C} 5$.

\section{Technical Note}

Results from cervical MRI (Fig. 1a) revealed a severe narrowing of the spinal canal at the levels of C3-C4 and C4$\mathrm{C} 5$ with compression extending behind $\mathrm{C} 4$ and $\mathrm{C} 5$. Intramedullary signal abnormality at $\mathrm{C} 3-\mathrm{C} 4$ and $\mathrm{C} 4-\mathrm{C} 5$ was present. Particularly, axial T2 MRI sequences (Fig. 1b-e) showed an important anterior compression at both these levels with compression of the spinal cord. The prevailing anterior compression was well demonstrated on CT scan with the presence of OPLL that extends in both the cranial and caudal direction with respect to the C4-C5 intervertebral disc, particularly behind the $\mathrm{C} 5$ body (Fig. 2a and b). Given the progressive neurological deterioration and the prevailing anterior compression, an anterior surgical procedure was proposed. At each level, under microscopic view, an accurate discectomy was performed, and selective drilling of the posteroinferior part of the overlying vertebrae and of the posterosuperior of the underlying one was performed in oblique fashion, drawing the shape of reversed wedge on the sagittal plane. A part of the OPLL was carefully removed, leaving in situ the part that was tightly attached to the dura mater. Lastly, two cages fixed with body screws (Zero-P VA, DePuy Synthes) were positioned in place of the removed discs, thus improving spinal stability and promoting bone fusion (Fig. 3). Results from the postsurgical CT scan showed an adequate decompression of the spinal cord and nerve roots with correct positioning of the cages and fixing screws (Fig. 4a and b). At 15 months, dynamic cervical Xrays (Fig. 5) demonstrated a solid fusion, and cervical MRI showed decompression but without restoration of CSF flow (Fig. 5).

\section{Discussion}

The surgical procedure for spinal cervical canal stenosis inducing myelopathy might be quite challenging. Once the surgery is proposed, the first step for the spine surgeon is to choose the most suitable surgical procedure for the single 


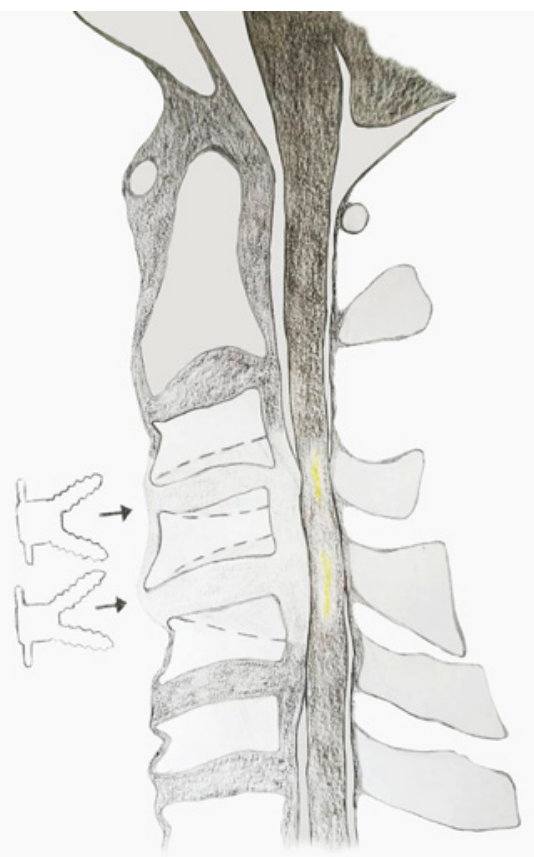

Figure 3. Schematic design of the surgical procedure in the sagittal plane. Spinal canal decompression at the end of selective somatectomy is shown in light gray color. Interrupted dots show the selective corpectomy extension at $\mathrm{C} 3, \mathrm{C} 4$, and $\mathrm{C} 5$. Intervertebral cages are also designed with arrows showing the direction of positioning. case. Anterior approaches are preferred in patients with anterior compression like osteophyte formation or discal herniations. Another indication for an anterior route approach is the presence of cervical kyphosis. Generally, anterior approaches are preferred in younger patients and in single level pathology. Posterior approaches are preferred in presence of multiple level stenosis and of posterior spinal cord compression by ligamentum flavum and when cervical spine lordosis is maintained.

Anterior cervical discectomy and fusion (ACDF) and anterior cervical corpectomy and fusion (ACCF) are the most popular and used anterior techniques. Some authors have described an oblique corpectomy in selected patients ${ }^{5}$.

ACDF is suitable for single or multiple levels of anterior spinal stenosis with osteophytic formation localized at the level of intervertebral disc without significant cranio-caudal extension. One relevant limitation of this technique is the removal of osteophytes extending above or below the intervertebral disc. In cases of osteophytes extending over the intervertebral disc or in cases where there is presence of calcification of the posterior longitudinal ligament, an ACCF might be necessary. Wide corpectomies permit an adequate vertical and horizontal decompression. One of the risks of multiple corpectomies is graft dislodgement ${ }^{6}$.

We report a modified anterior discectomy with selective posterior corpectomy performed instead of classical double corpectomy. Had we chosen a classical multiple corpectomy to obtain an optimal decompression, we would have had to
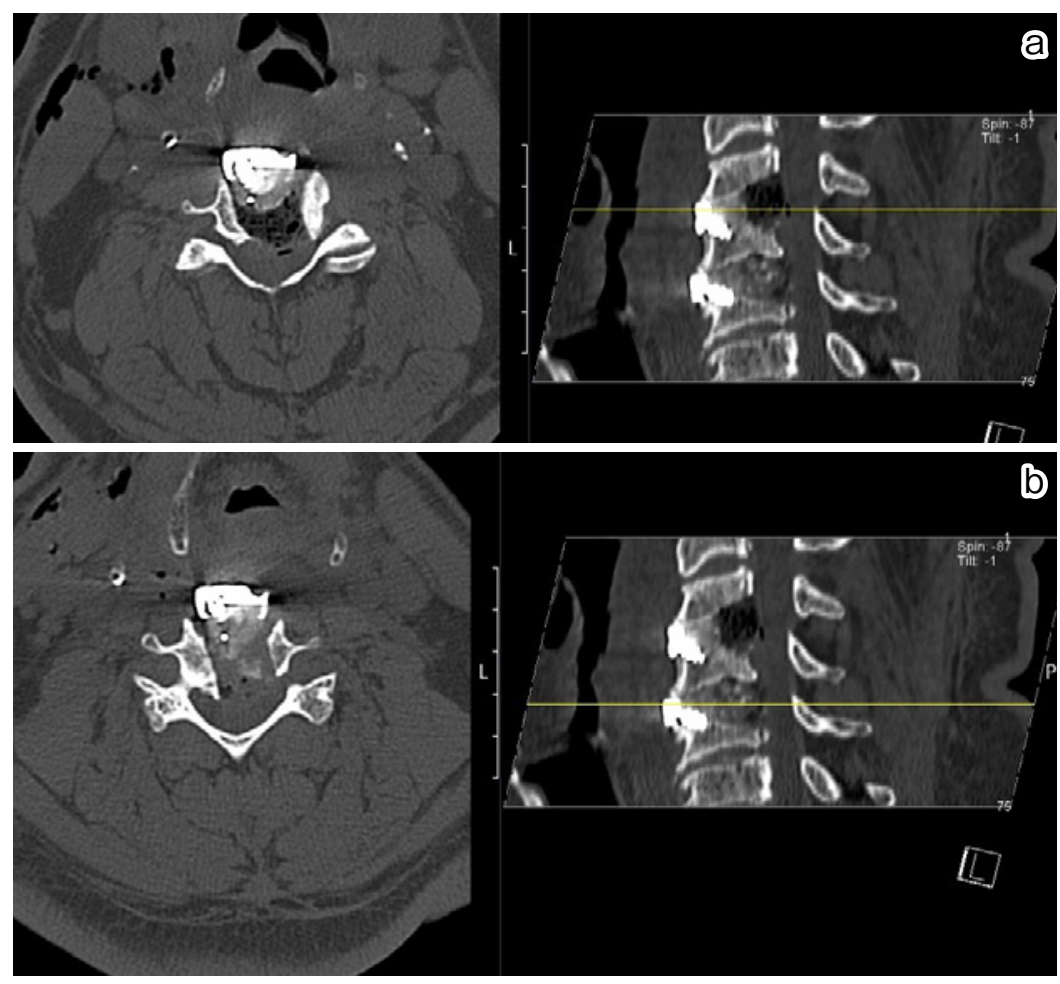

Figure 4. a. Axial CT scan images and sagittal reconstruction at the $\mathrm{C} 3-\mathrm{C} 4$ level showing the wide decompression medial to lateral and cranial to caudal. b. Axial CT scan images and sagittal reconstruction at the C4-C5 level showing the wide decompression medial to lateral and cranial to caudal extension, with minimal persistence of pre-foraminal osteophytes on the right side. 
perform a corpectomy of $\mathrm{C} 4$ and $\mathrm{C} 5$ with the need to place an expandable cage from $\mathrm{C} 3$ to $\mathrm{C} 6$ with anterior plating. A double level ACDF might have been inadequate due to the need to decompress not only behind the vertebral bodies but also at the level of the disc. A more selective resolution of the problem appeared possible, so we planned a $\mathrm{C} 3-\mathrm{C} 4$ and C4-C5 discectomy associated with posterior selective corpectomy of $\mathrm{C} 4$ and $\mathrm{C} 5$, removing part of the OPLL extending behind the vertebral body levels. With our procedure, we spared the C5-C6 disc and shifted this more challenging and potentially hazardous procedure to a modified double ACDF. The postsurgical cervical CT scan showed the decompression and the correct position of the cages. Thus, the early primary goal of spinal canal decompression was achieved. At 15 months of follow-up, dynamic X-rays showed a solid fusion (Fig. 6). Thus, the late primary goal of arthrodesis was achieved. Cervical MRI, beside its low quality, did not show restoration of CSF leak (Fig. 7). We suppose that this is due to the remaining tight adherent posterior ligament.

\section{Conclusion}

In recent years, reducing the invasiveness of single surgical procedures has become a major goal of spinal surgeons. Thus, we need to tailor the surgical procedure to each patient to reduce invasiveness while achieving the primary

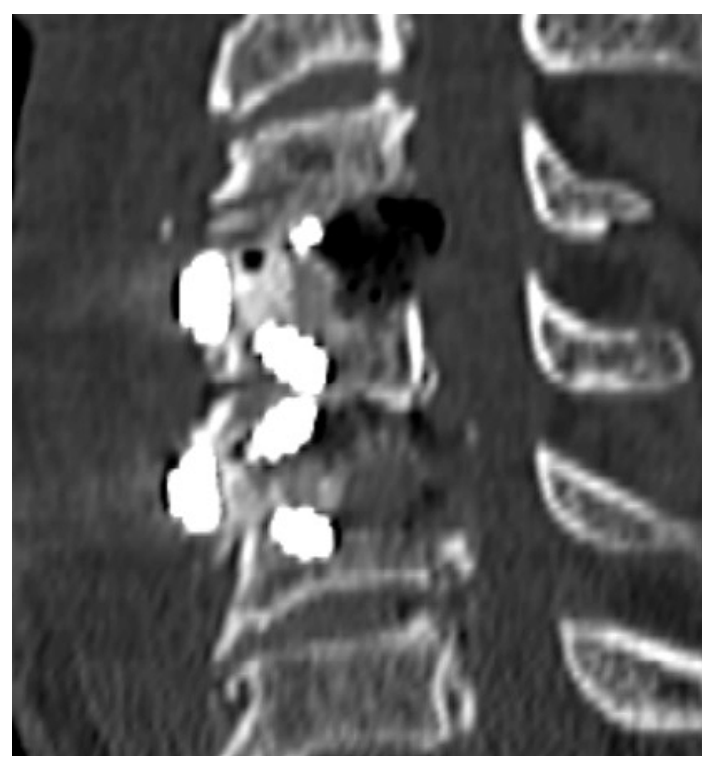

Figure 5. Postoperative sagittal reconstruction of CT scan showing the selective posterior corpectomy, removal of a part of ossification of the posterior longitudinal ligament, and cage positioning.

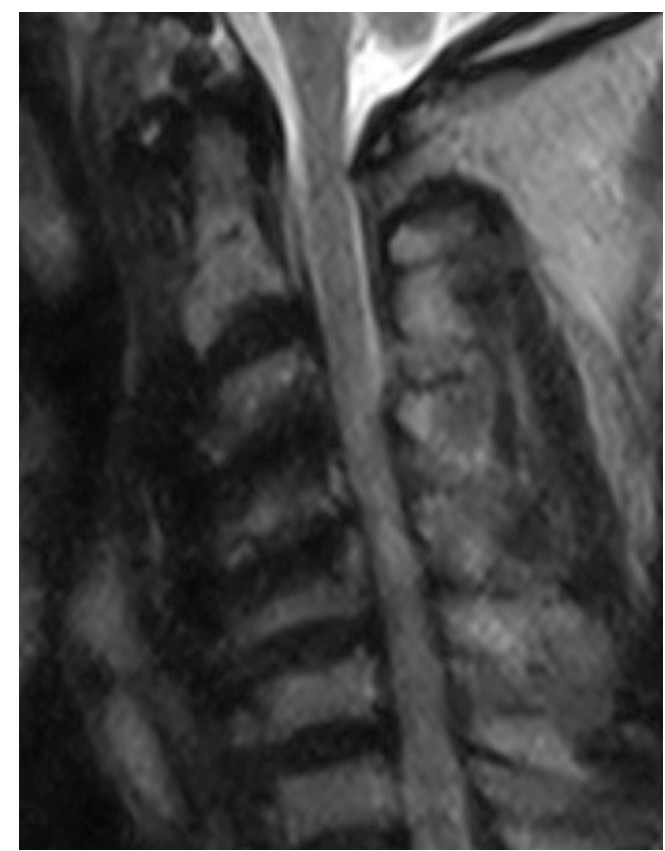

Figure 7. Sagittal T2 MRI images showing anterior decompression.

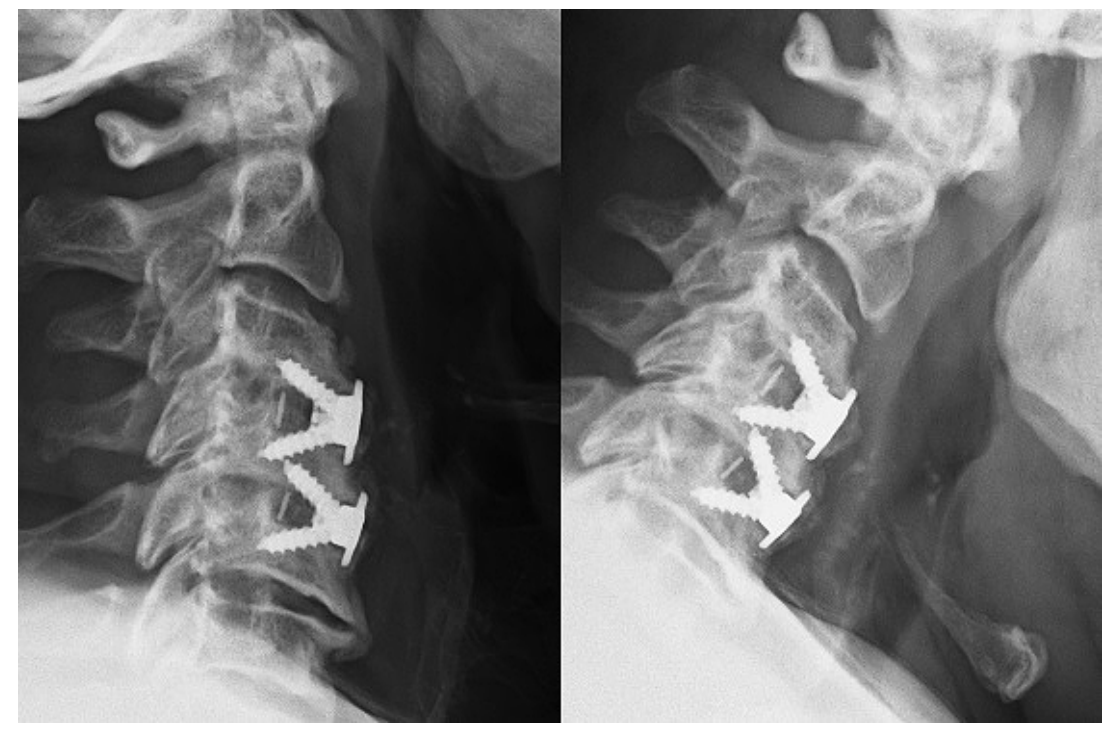

Figure 6. Flexion-extension $\mathrm{X}$-rays showing the stability at $\mathrm{C} 3-\mathrm{C} 4$ and $\mathrm{C} 4-\mathrm{C} 5$ and bone fusion. 
goals. Selective posterior somatectomy might be considered safe for ACDF as it potentially has less device problems compared with multiple corpectomies ${ }^{7}$. This procedure might be considered for patients with anterior spinal cord compression extended above and/or below the intervertebral disc space. Further studies with long follow-up times are necessary to confirm the safety and efficacy of our described procedure.

Conflicts of Interest: The authors declare that there are no relevant conflicts of interest.

Ethical Approval: This article does not contain any studies with human participants performed by any of the authors.

Informed Consent: The patient consented to the surgery and to have his data published.

\section{References}

1. Young WF. Cervical spondylotic myelopathy: a common cause of spinal cord dysfunction in older persons. Am Fam Physician.
2000;62(5):1064-70, 1073

2. Baron EM, Young WF. Cervical spondylotic myelopathy: a brief review of its pathophysiology, clinical course, and diagnosis. Neurosurgery. 2007;60(1 Supp1 1):S35-41.

3. Aljuboori Z, Boakye M. The Natural History of Cervical Spondylotic Myelopathy and Ossification of the Posterior Longitudinal Ligament: A Review Article. Cureus. 2019;11(7):e5074.

4. Ghogawala Z. Anterior cervical option to manage degenerative cervical myelopathy. Neurosurg Clin N Am. 2018;29(1):83-9.

5. Bruneau M, Cornelius JF, George B. Multilevel oblique corpectomies: surgical indications and technique. Neurosurgery. 2007;61(3 Suppl):106-12; discussion 112. Review.

6. Lin Q, Zhou X, Wang X, et al. A comparison of anterior cervical discectomy and corpectomy in patients with multilevel cervical spondylotic myelopathy. Eur Spine J. 2012;21(3):474-81.

7. Chen TP, Qian LG, Jiao JB, et al. Anterior decompression and fusion versus laminoplasty for cervical myelopathy due to ossification of posterior longitudinal ligament: A meta-analysis. Medicine (Baltimore). 2019;98(1):e13382.

Spine Surgery and Related Research is an Open Access journal distributed under the Creative Commons Attribution-NonCommercial-NoDerivatives 4.0 International License. To view the details of this license, please visit (https://creativeco mmons.org/licenses/by-nc-nd/4.0/). 